\title{
Investigation of the de vacuum breakdown mechanism
}

\author{
A. Descoeudres, Y. Levinsen, S. Calatroni, ${ }^{*}$ M. Taborelli, and W. Wuensch \\ European Organization for Nuclear Research, CERN, 1211 Geneva 23, Switzerland
}

(Received 14 April 2009; published 29 September 2009)

\begin{abstract}
Breakdowns occurring in rf accelerating structures will limit the ultimate performance of future linear colliders such as the Compact Linear Collider (CLIC). Because of the similarity of many aspects of dc and rf breakdown, a dc breakdown study is underway at CERN to better understand the vacuum breakdown mechanism in a simple setup. Measurements of the field enhancement factor $\beta$ show that the local breakdown field is constant and depends only on the electrode material. With copper electrodes, the local breakdown field is around $10.8 \mathrm{GV} / \mathrm{m}$, independent of the gap distance. The $\beta$ value characterizes the electrode surface state, and the next macroscopic breakdown field can be well predicted. In breakdown rate experiments, where a constant field is applied to the electrodes, clusters of consecutive breakdowns alternate with quiet periods. The occurrence and lengths of these clusters and quiet periods depend on the evolution of $\beta$. The application of a high field can even modify the electrode surface in the absence of breakdown. Measurements of time delays to breakdown show two distinct populations, immediate and delayed breakdowns, indicating that two different mechanisms could exist. The ratio of these two populations depends on the conditioning state of the electrodes and on material. Gas release during breakdown is dominated by $\mathrm{H}_{2}$ and $\mathrm{CO}$. This degassing is mainly due to electron-stimulated desorption. During the quiet periods without breakdown, gases are also released but the quantities are much smaller. All the measurements presented here emphasize the crucial role of field emission in the breakdown triggering.
\end{abstract}

DOI: 10.1103/PhysRevSTAB.12.092001

PACS numbers: 52.80.Vp, 79.70.+q, 52.80.Pi, 29.20.Ej

\section{INTRODUCTION}

The phenomenon of dc electrical breakdown between two metallic electrodes located in high vacuum has been studied for more than 50 years [1-8], but a complete understanding of this phenomenon is still lacking. The emission of electrons from the cathode by field emission (FE) indisputably seems to be a necessary stage in the breakdown process, acting either as a direct cause of breakdown or as a precursor for other secondary effects. Electron currents are emitted under the effect of the electric field from microscopic protrusions at the cathode surface. This FE current produces, for instance, a resistive heating of the protrusions. Depending on the current density, some gas will be desorbed or the protrusions could melt and even be locally evaporated. Then, the ionization of this vapor by the field emitted electrons could be the origin of the breakdown process. In this scenario, the creation of a microscopic gaseous medium is crucial for the development of the breakdown phenomenon in vacuum. Breakdowns could also be triggered by some other mechanisms involving the FE current: direct creation of a microplasma by the explosive evaporation of a strongly heated cathode protrusion, gas desorption at the anode caused by an intense FE current, or melting of a spot at the anode by a heavy bombardment of FE electrons.

*sergio.calatroni@cern.ch
Macroparticles released from the electrodes by fieldinduced stresses are also mentioned in the literature as a possible cause of breakdown, because they could be partly evaporated by the FE current [4]. Direct field evaporation of surface atoms is another possible mechanism of breakdown.

Since the field emission current is so important in the breakdown trigger, it is useful to describe the ability of a surface to emit electrons by FE by considering the field enhancement factor $\beta$. This factor is defined by the ratio between the local microscopic electric field seen at the emitting protrusion, and the macroscopic applied electric field. It is believed to be strongly related to the microscopic topography of the surface (roughness), since the field is more enhanced if very sharp protrusions exist. However, the nature of emitters with large $\beta$ values is not clear. For example, geometrical protrusions with $\beta>50$ have never been observed on typical scanning electron microscope images of copper surfaces. On a real surface, many field emitters are present with distributed $\beta$ values, the ones with the largest $\beta$ contributing the most to the emitted current. The factor $\beta$ can be analytically calculated through electrostatics for a single protrusion of simple geometry, such as a cone, for example [5]. The relationship between the applied field and the FE current density is given by the Fowler-Nordheim equation, in which $\beta$ appears as a sensitive parameter $[9,10]$. The measurement of $\beta$ is thus very important to understand results of break- 
down experiments. The work function of the electrode material is also another important parameter affecting the FE current.

For most of the materials, the breakdown field reached with new electrodes can be progressively raised by producing repetitive breakdowns between them. This conditioning process is believed to be related to modifications of the electrode surface state due to sparks: melting of fieldemitting protrusions, smoothing, cleaning, removal of surface oxides, and adsorbed impurities, for example $[7,8]$.

Vacuum breakdowns are studied and occur in different contexts, such as vacuum circuit breakers [11,12] and more recently in dc guns for $\mathrm{x}$-ray free electron lasers [13]. They are also of particular interest in $\mathrm{rf}$ accelerating structures [14-16], especially for future linear colliders such as the Compact Linear Collider (CLIC) [17,18]. In order to limit this linear collider to an acceptable length, extremely high accelerating gradients of the order of $100 \mathrm{MV} / \mathrm{m}$ are required, with corresponding macroscopic surface fields in excess of $200 \mathrm{MV} / \mathrm{m}$. With such fields, rf breakdowns occur and produce damage on the accelerating cavities. Furthermore, breakdowns during operation can lead to the loss of the accelerated beam due to random kicks. Thus, it is important to limit the occurrence of breakdowns inside the accelerating structures as much as possible, assuring that each structure has a very low breakdown probability (or breakdown rate). On the other hand, breakdowns are beneficial during an initial phase, because they condition the structures and improve their ability to sustain high gradients [19-21].

Because of the similarity of many aspects of dc and $\mathrm{rf}$ breakdown, a dc breakdown study is underway at CERN for the CLIC feasibility study. The dc tests are faster, more flexible, and are more easily instrumented than high power rf tests, and can provide benchmarking for simulation. The breakdown fields of several electrode materials and surface preparations have already been measured in a dc setup [2226]. The present article is focused on the mechanisms of breakdown and on the parameters governing it.

Measurements of the dc breakdown field and of the field enhancement factor obtained with copper electrodes are presented in Sec. III A. In analogy to rf tests, these values are measured in a "conditioning mode" where the field is increased until breakdown is reached, but also in a "breakdown rate mode" where the working field is kept constant. Besides these basic experiments, additional information about the breakdown mechanism is obtained by measuring the time delay before a breakdown, and by the analysis of the outgassing before and during a breakdown. These results are presented in Secs. III B and III C respectively.

\section{EXPERIMENTAL SETUP}

Figure 1 shows a schematic view of the dc spark setup. Both electrodes are made of pure copper (Cu OFE, UNS C10100) unless explicitly specified, in a point-to-plane

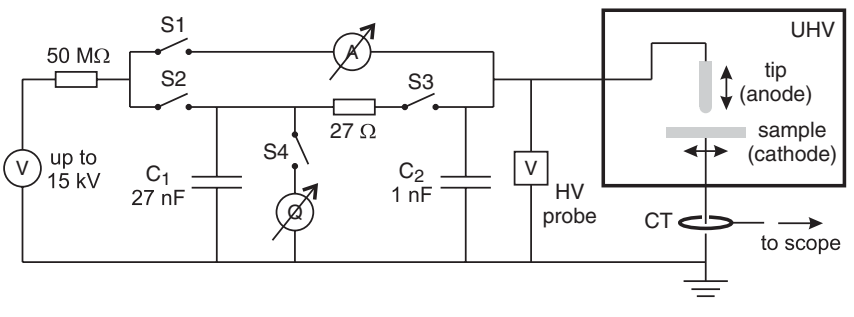

FIG. 1. Schematic drawing of the experimental setup.

configuration. They are located in an ultrahigh vacuum (UHV) chamber with a typical pressure of $5 \times$ $10^{-10}$ mbar. When bakeouts are performed to reach $\mathrm{UHV}$, the vacuum chamber is typically heated to $150^{\circ} \mathrm{C}$ for 24 hours. The anode is a hemispherical rounded tip, $2.3 \mathrm{~mm}$ in diameter, and the cathode (sample) is a grounded $10 \mathrm{~mm} \times 50 \mathrm{~mm}$ rectangular plane surface, $2 \mathrm{~mm}$ in thickness. Samples are directly cut from polycrystalline cold rolled sheets and tips are obtained by turning the end of cylindrical rods. Unless explicitly specified, no preliminary heat treatments are applied to the electrodes. Both electrodes are cleaned according to the CERN standard procedure for UHV components [27] prior to installation in the UHV chamber. The sample can be moved laterally inside the chamber in order to test several spots on its surface. The vertical position of the tip, and therefore the gap distance, is controlled with a calibrated micropositioning device combined with differential levers. The positioning accuracy of this system is around $1 \mu \mathrm{m}$ and the gap distance is set between 10 to $50 \mu \mathrm{m}$, with a typical distance of $20 \mu \mathrm{m}$. Such small gaps are necessary to reach fields of several hundred $\mathrm{MV} / \mathrm{m}$ with the available $15 \mathrm{kV}$ power supply. The zero distance is found by bringing the electrodes into contact and measuring a short circuit.

Field emission measurements between the electrodes are performed by closing the $S 1$ relay and by applying high voltage to the anode directly from the power supply. The FE current is read with a multimeter. The voltage is increased from zero to the value producing a FE current of $10^{-7} \mathrm{~A}$, which is a safe value. With such a low FE current, a breakdown is not triggered and damaging the multimeter is avoided. From these current-voltage characteristics, and the assumption that they follow a Fowler-Nordheim behavior, the field enhancement factor $\beta$ and the emitting area can be calculated [22]. FE current data can be well fitted between $10^{-11}$ and $10^{-8} \mathrm{~A}$ with Fowler-Nordheim curves (regression correlation coefficient >0.99). Assuming that the emitting area is circular, its diameter is typically measured to be between 20 to $80 \mathrm{~nm}$. Since the variations in FE current are much larger, they are mainly the result of changes in $\beta$. The local microscopic electric field at the surface is then given by the macroscopic electric field multiplied by $\beta$.

For the measurement of the breakdown field $E_{b}$, the $27 \mathrm{nF}$ capacitor $C_{1}$ is charged with the power supply first to a low value via the relay $S 2$, and then connected to the 
anode via the high current relay $S 3$ for typically 2 seconds. If no breakdown occurs, the voltage is increased and the cycle is repeated until a breakdown occurs. Sparks are repeated in order to condition the tested spots on the electrode surfaces. The accuracy of the gap distance is checked before and several times during a conditioning experiment by reestablishing contact between the two electrodes. Breakdowns are detected either by measuring the remaining charge on the capacitor after the exposure time by closing the relay $S 4$, or simply with a $500 \mathrm{MHz}$ current transformer (CT) connected to a $1 \mathrm{GHz}$ scope. The current transformer detects currents above $1 \mathrm{~A}$. The $C_{2}$ capacitor is used to damp voltage overshoots when the $S 3$ switch is closed. For breakdown rate (BDR) measurements, the main capacitor $C_{1}$ is always charged at the same voltage and then connected to the anode for one second. If a breakdown occurs during this period, it is detected with the current transformer. The breakdown rate is defined as the fraction of attempts in which a breakdown occurs. In the present setup, the maximum energy available for the discharge is around $1 \mathrm{~J}$ and was chosen to be of the same order as the total available energy in the rf experiments at $30 \mathrm{GHz}$ conducted at CERN. More details about the setup can be found in [22].

Time delays between the voltage rise and a breakdown were measured with a $75 \mathrm{MHz}$ high voltage probe, also connected to the scope. Typical FE currents before breakdown are in the $\mu \mathrm{A}$ range, too low to significantly discharge the main capacitor $C_{1}$ during one pulse. Thus, the applied voltage remains constant during the pulse, as it is verified with the voltage probe. The composition of gases released by breakdowns were identified with a residual gas analyzer (RGA) connected to the vacuum chamber. Quantitative measurements are also possible in our system, since the pumping speed has been measured and the relation between the partial pressure of a gas (measured with a vacuum gauge) and the corresponding ion current signal (measured with the RGA) has been calibrated. The quantity of gas released by a breakdown can thus be calculated by integrating the time-resolved ion current signal. Since the amount of released gas per breakdown is relatively small, the pumping speed is reduced during such measurements down to $0.02 \mathrm{l} / \mathrm{s}\left(\mathrm{N}_{2}\right.$ equivalent) by pumping through a small diameter by-pass pipe. In this way, ion current signals do not decay too quickly and can be integrated more precisely.

\section{RESULTS AND DISCUSSION}

\section{A. Breakdown field and field enhancement factor measurements with copper electrodes}

The evolution of the breakdown field $E_{b}$ and the field enhancement factor $\beta$ during a typical conditioning experiment with copper electrodes is shown in Fig. 2. Measurements of $\beta$ and of $E_{b}$ are made alternatively. Because of erosion and material displacement, the gap



FIG. 2. Evolution of the field enhancement factor $\beta$ and the subsequent breakdown field $E_{b}$ with $\mathrm{Cu}$ electrodes during a conditioning experiment.

distance varies slightly during the experiment (increases or decreases). After 50 breakdowns, variations are typically less than $\pm 10 \%$ of the original gap distance. All values of $E_{b}$ and $\beta$ presented in this paper are corrected taking these changes in the gap distance into account. Contrary to other materials [26], a conditioning phase is not observed with copper electrodes. The first values of the breakdown field are generally significantly higher than the average of all values $\bar{E}_{b}$. This is due to the good surface state of the electrodes before the start of the sparking experiment. The first $\beta$ value before any spark is indeed generally low, around 10, indicating that electrodes surfaces are smooth. Then the electrodes are eroded by the sparks and the roughness increases (postexperiments scanning electron microscope pictures can be found in [23]). As a consequence $\beta$ also increases after a few sparks, so the FE current is higher for a given field. This results in a decrease in the breakdown field as can be seen in Fig. 2. The decrease in $E_{b}$ is possibly due to the energy released by one spark (around $1 \mathrm{~J}$ ), which might be too large and produce damage on electrodes. Tests with lower energies are underway in order to verify this assumption.

The first phase where the breakdown field decreases and the field enhancement factor increases generally lasts only a very few breakdowns, often only one. A more stable situation is reached afterwards, during which $E_{b}$ and $\beta$ vary roughly within $35 \%$ around their average value (typically $\sim 160 \mathrm{MV} / \mathrm{m}$ and $\sim 75$, respectively). This scattering of the data is caused by continuous surface modifications due to sparks, leading either to improved or worsened resistance to breakdown.

The effects of different surface treatments of copper electrodes have been reported previously [26]. It has been found that the different treatments only affect the breakdown field and the field enhancement factor for the 
very first breakdowns. After that, the benefit of a careful surface preparation is rapidly erased by the first sparks and all samples behave in a similar way. This is more evidence that the high breakdown field observed for the first breakdowns in Fig. 2 is related to the good initial surface state of the electrodes.

It is clear from Fig. 2 that $E_{b}$ and $\beta$ do not behave independently of each other, since the two curves are roughly symmetric. The correlation can be seen better in Fig. 3(a), where each $\beta$ value is plotted against the breakdown field directly following the measurement of $\beta$. If the electrodes have a high $\beta$ value at a given moment during the experiment, the following breakdown will generally occur at a low field due to enhanced field emission. Inversely, the breakdown field will be high if the electrodes are in a state with a low $\beta$ value. The same correlation is also observed with molybdenum electrodes. The electrodes used in these experiments are made of $99.95 \%$ purity Mo, prepared in the same way as $\mathrm{Cu}$ electrodes except for an additional chemical etching to remove surface oxides.

Whereas a strong correlation exists between $\beta$ and the next breakdown field, it is not the case for $\beta$ and the previous breakdown field. The data of Fig. 3(b) are indeed much more scattered. In other words, the surface state resulting after a breakdown is not clearly determined by the value of the preceding breakdown field. For example, a breakdown occurring at a high field will not necessarily produce a rough surface, and therefore a high $\beta$. One sees in Fig. 3(b) that a breakdown occurring at $200 \mathrm{MV} / \mathrm{m}$ can produce $\beta$ values as low as 30 and up to 140 .

Data of Fig. 3(a) can well be fitted with

$$
\beta \cdot E_{b}=\text { constant }
$$

indicating that the local microscopic breakdown field, which is precisely equal by definition to the breakdown field multiplied by the field enhancement factor, is constant throughout the whole experiment. A breakdown is thus triggered as soon as the local electric field reaches a critical threshold. Consequently, the breakdown field can be well predicted at any moment if the present $\beta$ value of the electrodes is known. This result is in agreement with previous measurements, where the local breakdown field is claimed to be only dependent on the electrode material $[2,28]$.

The evolution of the local breakdown field during a typical conditioning experiment is given in Fig. 4. One can clearly see that the values of the local breakdown field are much less dispersed than those of $E_{b}$ and $\beta$, which supports the conclusion that $\beta E_{b}$ is roughly constant in these experiments. In particular, the breakdown field $E_{b}$ has a mean of $159 \mathrm{MV} / \mathrm{m}$ and a standard deviation of $32 \%$, the field enhancement factor $\beta$ has a mean of 77 and a standard deviation of $36 \%$, and the local breakdown field $\beta E_{b}$ has a mean of $10.8 \mathrm{GV} / \mathrm{m}$ and a standard deviation of $16 \%$. This value for the local breakdown field of copper is consistent with other experiments [28].

The arrows in Fig. 4 mark the values for the first breakdown, which are significantly different from the others as previously noted. The initial phase where $E_{b}$ decreases and $\beta$ increases lasts only for two breakdowns in this example. Even when $E_{b}$ is strongly decreasing, it is nevertheless interesting to note that the local breakdown field is increasing from $5 \mathrm{GV} / \mathrm{m}$ to higher values during this first phase. Therefore this can be viewed as a real conditioning phase, during which the local breakdown field is improved by the consecutive sparks. Thus, the physical quantity evolving and improving during the conditioning process in such experiments seems to be the local breakdown field rather than simply the breakdown field.

The influence of the gap distance between the electrodes on the average values of $E_{b}, \beta$, and $\beta E_{b}$ is presented in Fig. 5. Whereas the breakdown field and the field enhancement factor depend clearly on the gap, the local breakdown field remains constant around its value of $10.8 \mathrm{GV} / \mathrm{m}$. The field enhancement factor has the tendency to increase with the gap distance, because in a point-to-plane geometry the number of field emitters contributing to the total FE current is statistically increased if the gap distance is increased. As it can be seen in Fig. 6, the field on the cathode plane is indeed always decreasing with the distance from the sym-
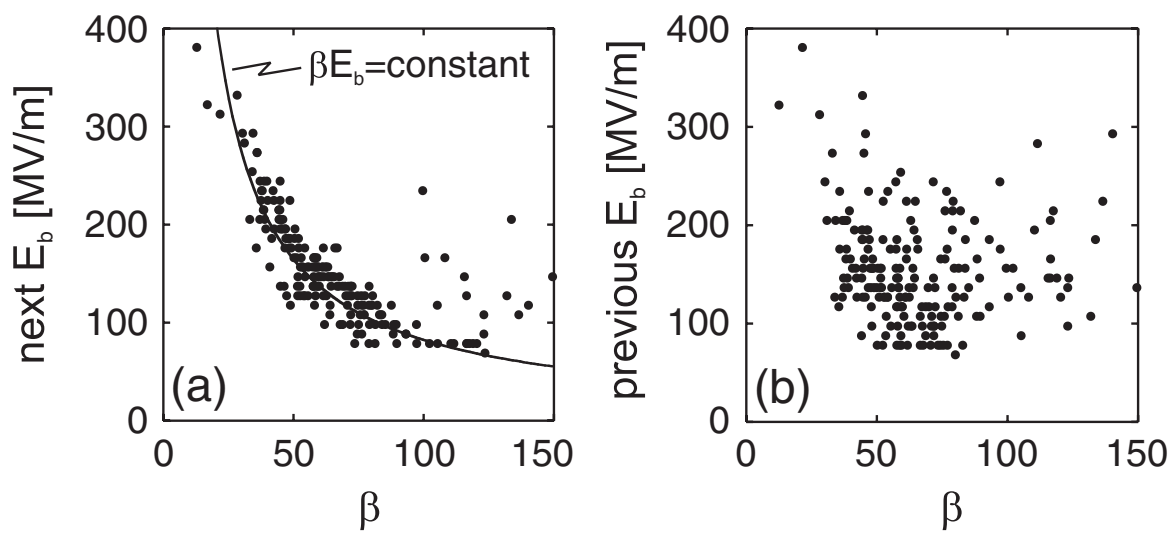

FIG. 3. Relation between the field enhancement factor $\beta$ with: (a) the next breakdown field; (b) the previous breakdown field. 


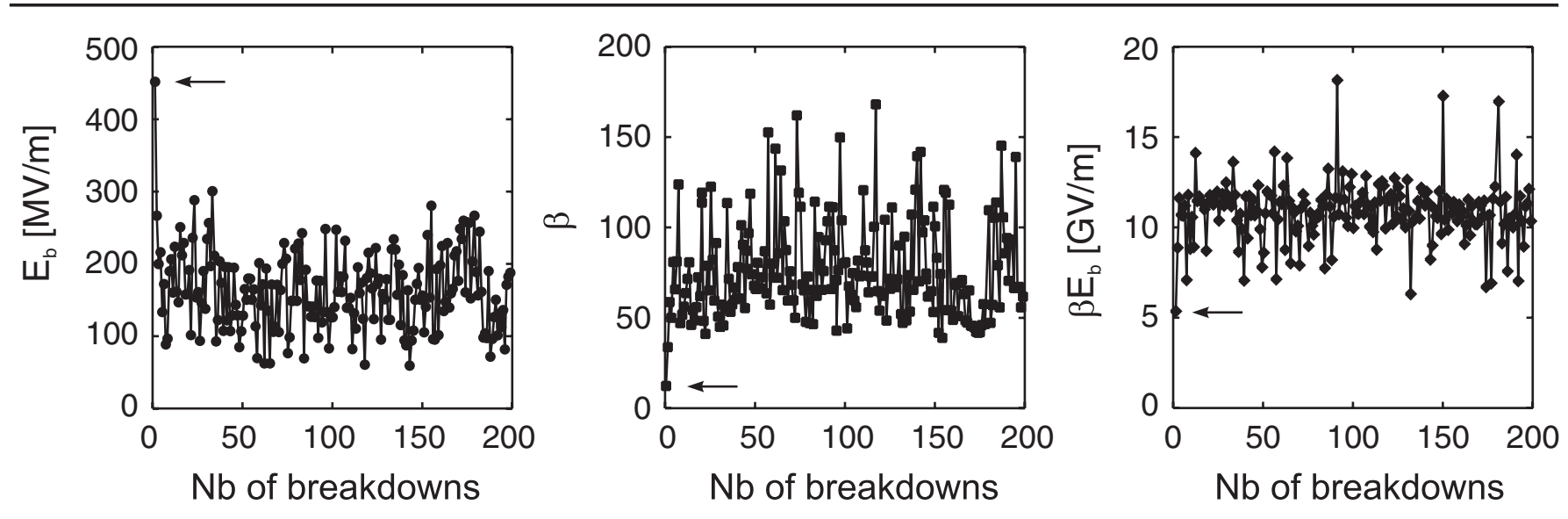

FIG. 4. Evolution of $E_{b}, \beta$ and local breakdown field during a typical conditioning experiment with Cu electrodes. Arrows indicate values of the first breakdown.

metry axis, but at a lower rate if the gap distance is large. Therefore, a larger surface area on the cathode will be exposed to the electric field with a larger gap distance. Because of this flattening of the field profile, the probability that an emitter with a larger $\beta$ value is exposed to the field also increases (many emitters are present on a real surface with distributed $\beta$ values). Since the shape of the FE current-voltage characteristic is dominated by the emitter with the largest $\beta$ present on the exposed area, the experimentally measured $\beta$ value will ultimately tend towards the maximum $\beta$ value present on the surface, if the gap distance is further increased.

This general behavior of $\beta$ with the gap distance is well described by a simple statistical model [29]. In this model, a certain number of field emitters are randomly distributed over the cathode surface, and each emitter is assigned a given $\beta$ value taken from a Gaussian distribution. The total FE current is calculated by summing the individual currents of each emitter, taking into account the local field induced by the macroscopic point-to-plane geometry and

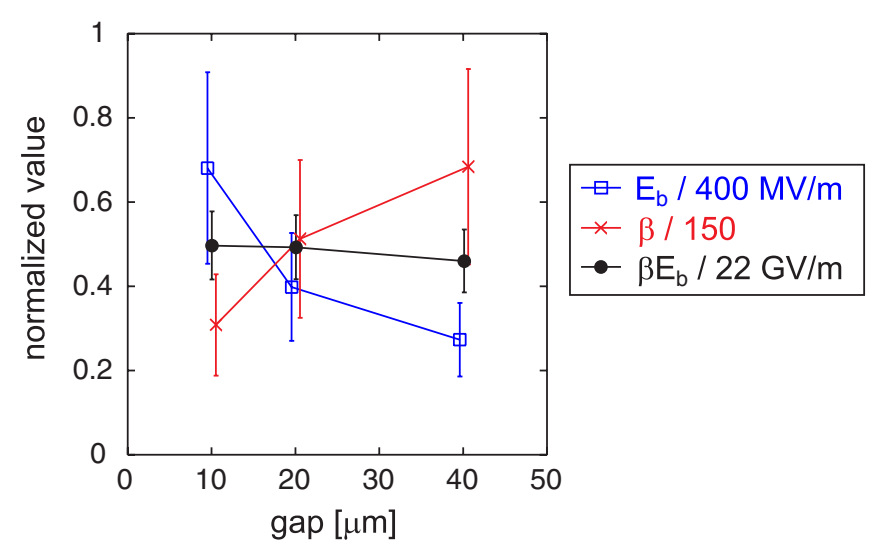

FIG. 5. (Color) Effect of the gap distance (10, 20, and $40 \mu \mathrm{m})$ on the average values of $E_{b}, \beta$ and of the local breakdown field. For clarity, values are normalized to $400 \mathrm{MV} / \mathrm{m}, 150$, and $22 \mathrm{GV} / \mathrm{m}$, respectively. assuming Fowler-Nordheim emission. The $\beta$ value which would be experimentally measured is deduced from this total FE current and the macroscopic field $V / d$, where $V$ is the voltage across the electrodes and $d$ the gap distance. If the emitter density on the surface is low enough, the measured $\beta$ can increase with the gap distance due to the limited sampling of the cathode area. For example, a calculation with 50 emitters distributed within a radius of $200 \mu \mathrm{m}$ reproduces a variation of the measured $\beta$ with the gap distance similar to what is experimentally observed in Fig. 5 [29]. In addition to this statistical effect, high $\beta$ values could also result from surface modifications induced by breakdowns during conditioning. Since the energy of a breakdown is higher with a higher gap distance for a given field, the induced surface damage is probably more severe and thus emitters with higher $\beta$ values could be created. Measurements of $\beta$ for gaps below $10 \mu \mathrm{m}$ have

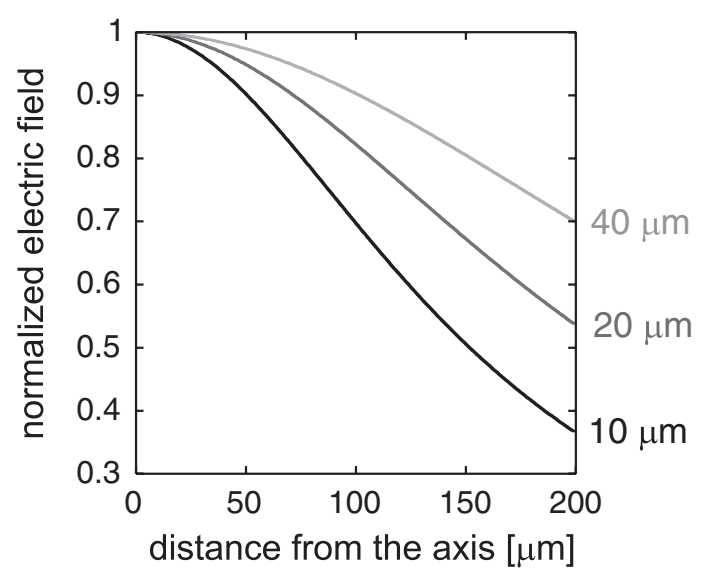

FIG. 6. Profiles of the electric field on the cathode surface, calculated for different gap distances. The anode is a hemispherical rounded tip, $2.3 \mathrm{~mm}$ in diameter, and the cathode is an infinite plane surface. The maximum value of the field corresponds to the parallel plate macroscopic field $V / d$, where $V$ is the voltage across the electrodes and $d$ the gap distance. 
been previously reported [1,2] and follow a similar trend as the one of Fig. 5. The decrease in $E_{b}$ with the gap is a direct consequence of the increase in $\beta$, since the local breakdown field is a constant value only dependent on the material and not on the geometry or gap spacing.

When working in breakdown rate mode, i.e., with the field kept at a fixed value during each attempt, breakdowns occur stochastically in time during the experimental run, but often come in groups. Clusters of consecutive breakdowns at each attempt are generally alternating with quiet periods, where no breakdown occurs. This feature is also observed in rf experiments, especially at a high breakdown rate [30]. Since the triggering of breakdowns is associated with field emission and therefore depends on the field enhancement factor, the evolution of $\beta$ is of particular interest during quiet periods in BDR mode. Figure 7 shows two examples of BDR runs, the first at a breakdown rate in the $10^{-3}$ range and the second in the $10^{-1}$ range. Measurements of $\beta$ are made after each attempt.

Unsurprisingly, $\beta$ remains low during quiet periods, typically below 50 . This is particularly clear in the long quiet period in Fig. 7(a), where $\beta$ decreases after a series of sparks and stabilizes around 40 . Since $\beta$ is not evolving, it indicates that the electrodes are in a stable state where the repetitive applications of the field seem to have no effect on their surfaces. On the other hand, it appears that $\beta$ is modified if the field is a bit higher. One observes in Fig. 7(b) that there is an upward drift of $\beta$ during all the quiet periods before clusters of breakdowns. Thus, the application of a sufficiently high field induces surface modifications on the electrodes, even without breakdown. A possible explanation of this is that a small protrusion is pulled out from the surface under the effect of the electrostatic force induced by the field (formation of Taylor cones [16,31]). The field enhancement factor is consequently slightly increasing during the quiet period, until it reaches roughly 50 which appears to be the threshold for this particular value of the working field. Since the local breakdown field is the same for every breakdown within the measured uncertainty margin, we have indeed

$$
\beta_{\text {threshold }}=\frac{\text { local breakdown field }}{\text { applied field }}=\frac{10.8 \mathrm{GV} / \mathrm{m}}{225 \mathrm{MV} / \mathrm{m}}=48 \text {. }
$$

Once this threshold is reached, a series of breakdown will occur. The surface modifications induced by the sparks result in strongly changing $\beta$ values, generally ranging from 50 to 150 . Breakdowns stop as soon as the electrodes are conditioned in a particular state characterized by a $\beta$ below $\beta_{\text {threshold }}$. Then a new quiet period starts, during which the field will slowly modify the surfaces and increase $\beta$ until it reaches the breakdown threshold again. The precise mechanism explaining why and how much $\beta$ is modified by the field is still unclear, but these coupled measurements of $\beta$ and $E$ are in good agreement with this simple scenario. This threshold is a consequence of the very steep dependence of the breakdown rate with the applied field, which is measured both in rf and dc experiments $[20,26]$. The threshold is thus the region where $\beta$ produces a local field which corresponds to a breakdown rate close to 1 .

The occurrence and duration of breakdown clusters and quiet periods observed in BDR experiments appear to be stochastic. We see here that the origin of this randomness is strongly related to the rather unpredictable evolution of $\beta$ after a breakdown [see Fig. 3(b)], or in other words to the variety of damage caused to electrodes by a spark. The duration of a quiet period will depend on how low $\beta$ has dropped below the threshold after the last breakdown of the preceding cluster (more time is needed to reach the threshold again if the starting value is very low), and probably also on the field level (the rising speed of $\beta$ seems to be higher with a higher field). Clearly, clusters of breakdowns statistically last longer at a high working field because (a) $200 \mathrm{MV} / \mathrm{m}$

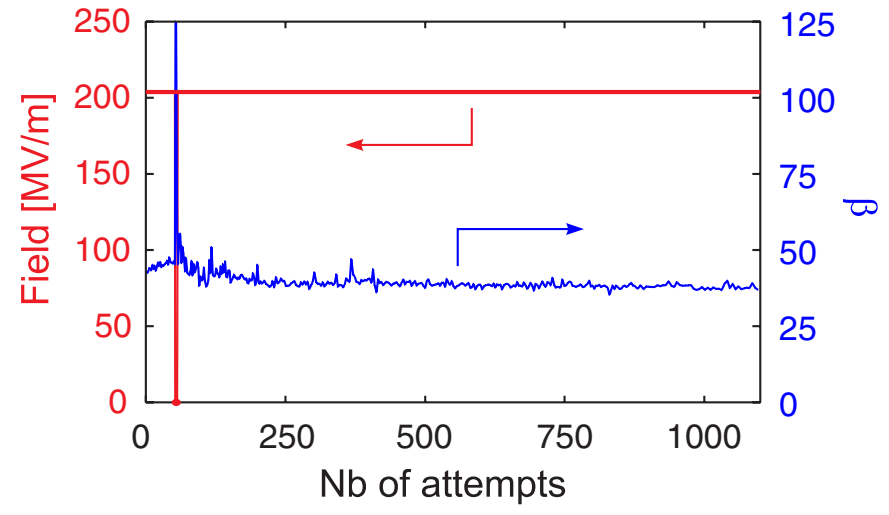

(b) $225 \mathrm{MV} / \mathrm{m}$

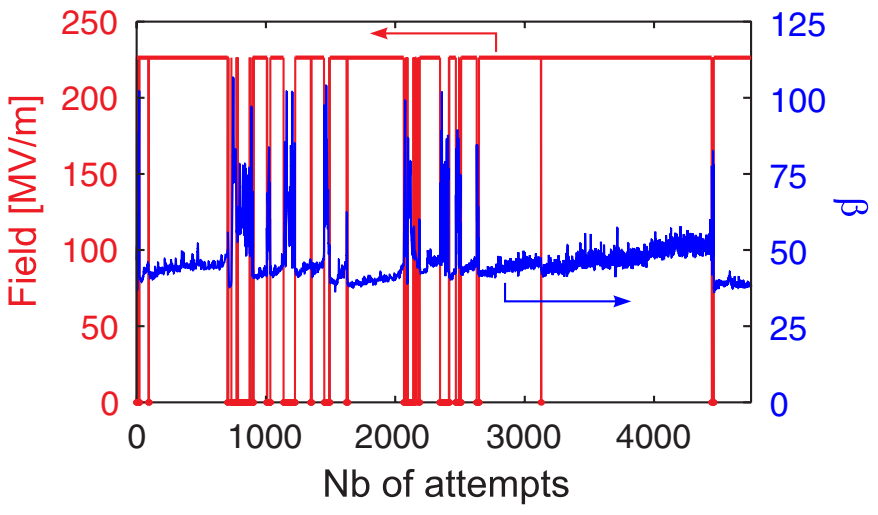

FIG. 7. (Color) Evolution of $\beta$ during breakdown rate measurements: (a) at $200 \mathrm{MV} / \mathrm{m}\left(\mathrm{BDR}=4 \times 10^{-3}\right)$; (b) at $225 \mathrm{MV} / \mathrm{m}$ $(\mathrm{BDR}=0.11)$. The electric field is in red, $\beta$ in blue. The electric field is plotted at zero if a breakdown occurs, at the working value otherwise. 


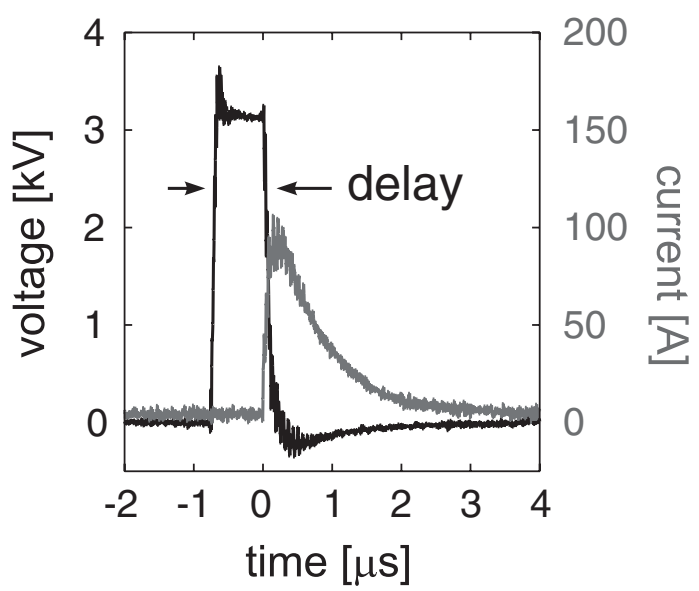

FIG. 8. Example of current and voltage measurements. The voltage is in black, the current in grey. The time delay before the breakdown is deduced from these signals. The time $t=0$ corresponds to the breakdown. A slight short overshoot in voltage is observed when the field is applied to the electrodes.

$\beta_{\text {threshold }}$ will be lower [see Eq. (2)]. Therefore, the probability that $\beta$ goes under the threshold after a breakdown strongly decreases. This effect is observed in real experiments, where the duration of breakdown clusters increases with the field (see Fig. 7). Finally, the breakdown probability reaches 1 when the working field is high enough, so that the lowest $\beta$ value that can possibly result after a breakdown is always above $\beta_{\text {threshold }}$.

\section{B. Time delays before breakdown}

The time delay between the application to the electrodes of the high voltage and the breakdown is an interesting parameter to study because the time scale of the breakdown process can possibly give some information about its mechanism [32,33]. Figure 8 shows a typical example of voltage and current measurements, and the time delay deduced from them. The instant of breakdown is characterized by the abrupt drop of the voltage and the rise of the current. The presence of a large current is possible at this moment, because a plasma has been created and the vacuum space between the electrodes loses its insulating properties. The shape of the current signal is directly related to the electric configuration of the setup. In our case, the spark is created by the discharge of a capacitor in an RC circuit (see Fig. 1). The typical duration of a spark is thus around $2 \mu \mathrm{s}$ in our setup. All the measurements of time delays presented here are made in the conditioning mode, i.e., with a small increase in the field (typically of the order of $5 \mathrm{MV} / \mathrm{m}$ ) at each attempt until breakdown is reached.

With $\mathrm{Cu}$ electrodes, breakdowns generally occur as soon as the nominal voltage is applied. But a few, about 5\%, occur with longer time delays. This effect is much clearer with molybdenum electrodes. Histograms of delays measured during a conditioning experiment are presented in Fig. 9. Two populations of delays are clearly visible, which could indicate two different breakdown mechanisms. The first, in black, has an average of $129 \mathrm{~ns}$ (standard deviation $\sigma=16 \mathrm{~ns}$ ). Since the rise time of the voltage in our setup has been measured to be around $100 \mathrm{~ns}$, breakdowns of this first population are "immediate." The second population of delays, in grey, has an average of $1.17 \mathrm{~ms}$ ( $\sigma=$ $0.33 \mathrm{~ms})$. These breakdowns will be called "delayed breakdowns," since the voltage holds constant for a certain amount of time. These long delays have probably no equivalent in CLIC rf tests since the typical time scale of one rf pulse is only $240 \mathrm{~ns}$. The mechanism of dc breakdown is probably more comparable to the one of rf breakdown in the $\mathrm{Cu}$ case than in the Mo case, because with $\mathrm{Cu}$ electrodes almost every breakdown is immediate. However, the pulse length dependence of breakdown ob-
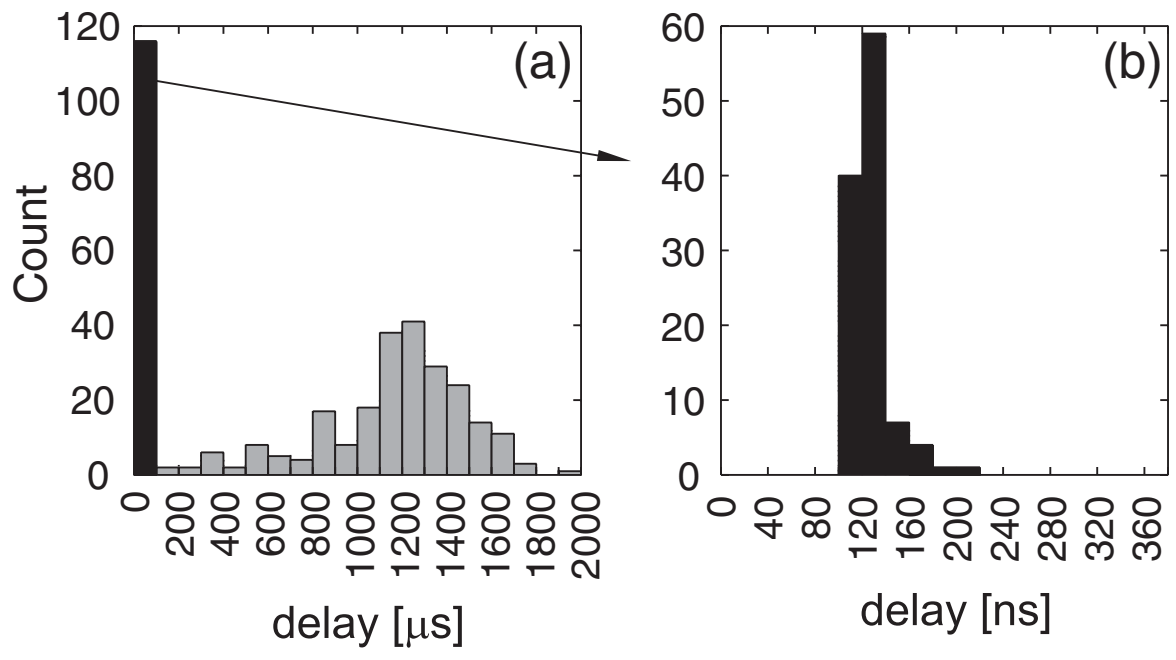

FIG. 9. Histograms of time delays with Mo electrodes during a conditioning experiment (350 breakdowns in total). Delays shorter than $1 \mu$ s are displayed in black. These short delays are plotted with a higher time resolution in (b). 
served in rf experiments [20] is not easily comparable with results of dc experiments.

It has been reported that vacuum breakdowns with delays shorter than $1 \mu \mathrm{s}$ occur preferentially when field emitters are the direct cause of the breakdown triggering [32], whereas the origin of breakdowns with longer delays in the ms range is much less clear. Such breakdowns could rather be related to secondary effects such as the release from the electrodes of droplets, clumps or macroparticles, or their impact on the electrodes [32,34]. The role of residual vapor in the electrode gap or gas desorbed from the electrodes has also been pointed out as a possible cause of breakdowns with very long delays [33]. The outgassing of the electrodes will be discussed in the next section.

In our experiments, breakdowns of both populations occur at every field, but immediate breakdowns dominate during the conditioning phase of the electrodes. For example, more than $80 \%$ of all breakdowns are immediate during the conditioning of Mo electrodes. On the other hand, delayed breakdowns dominate after the conditioning process $(75 \%$ of all breakdowns are delayed with Mo electrodes). This behavior is also observed with other materials, such as stainless steel, for example. Thus, immediate breakdowns are rather associated with a "weak" electrode surface state, whereas delayed breakdowns are associated to a surface state more resistant to breakdown. According to the studies cited above, the conditioning process would consist principally in removing the contaminants and the microscopic protrusions on the electrode surfaces which are the most efficient field emitters, because a majority of immediate breakdowns are measured in this phase. In agreement with this, $\beta$ values are observed to be slightly higher during the conditioning phase of Mo. Once the surfaces have been "cleaned" by the conditioning process, breakdowns are then rather caused by the ionization of some gas desorbed from the surfaces or by other secondary effects of the FE current, because delayed breakdowns dominate in this second phase. It should be noted that, even if a large number of efficient field emitters are destroyed during conditioning, the sparks leave craters on the electrode surfaces [23].

In breakdown rate mode with Mo electrodes, long delays are usually observed at the beginning of a cluster of breakdowns, typically for the very first spark, and also at the end of a cluster. This observation is consistent with the assumption that delayed breakdowns are related to a surface state somehow more resistant to breakdown. In BDR experiments, the first breakdown of a cluster is rather difficult to provoke, since a stable quiet period characterized by a low $\beta$ has immediately preceded. After this first breakdown, $\beta$ generally increases drastically which leads to immediate breakdowns. Towards the end of the cluster, the electrodes have also the tendency to return to a more stable state, which results finally in the following quiet period. Therefore, the presence of delayed breakdowns at the beginning and at the end of a cluster is in agreement with the results obtained in the conditioning mode.

The numeric distribution between immediate and delayed breakdowns depends on the material, as shown in Fig. 10. In addition to copper and molybdenum, tantalum (99.9\% purity) and stainless steel (316LN) were also tested with the same electrode preparation. The materials are ranked in this figure according to their average breakdown field, measured after the conditioning phase. The fraction $R$ of delayed breakdowns among all breakdowns (excluding the conditioning phase) is given in Table I for these different materials. It is observed that $R$ increases with the average breakdown field of the material, but the origin of this effect is still unclear.

\section{Outgassing before and during breakdown}

When a breakdown occurs, a short pressure rise is always observed in the vacuum chamber. The ratio between the pressure measured directly after a breakdown and the base pressure is typically between 10 and 50, depending mainly on the breakdown voltage. Analysis with a RGA shows that the main gases released are $\mathrm{H}_{2}, \mathrm{CO}, \mathrm{CH}_{4}$, and $\mathrm{CO}_{2}$. These gases are usually present in vacuum systems, since they are the typical species adsorbed on surfaces or formed by the impurities diffusing from the metal bulk. Figure 11 shows the average number of molecules of each gas released per breakdown, for different electrode materials.

The gas species and their partial ratios are not dependent on the electrode materials, which suggests that the dominant mechanism of gas release is electron-stimulated desorption (ESD) [35] from the anode surface. Indeed, the ratios found here are similar to those measured by ESD on stainless steel surfaces cleaned with the same procedure as for our electrodes [36]. Gases present in the bulk of the material can also be thermally released, since a large amount of energy is brought to the electrodes during a breakdown. It should be noted that there could also be other gases coming out of a spark, especially the bulk material itself. However, the probability that such heavy ions stick on the chamber walls (sticking factor) is close to one. Since the RGA is not in line of sight to the electrodes, these will not be measured.

The number of molecules released by Mo electrodes is a bit higher than that obtained from $\mathrm{Cu}$ electrodes, but this is probably mainly due to the different working voltage applied to the electrodes (a larger voltage has to be applied because the breakdown field of Mo is higher). In the case of copper, a slight reduction of gas release can be obtained if the electrodes are heat treated before the experiments. Here, $\mathrm{Cu}$ electrodes were heated ex situ during $2 \mathrm{~h}$ at $815^{\circ} \mathrm{C}$ in a UHV furnace (pressure below $5 \times$ $10^{-7} \mathrm{mbar}$ ), and then mounted into the spark test chamber. An outgassing of the electrodes occurs during this preliminary treatment, and therefore the number of molecules released during breakdowns is slightly reduced. 
(a) $\mathrm{Cu}$

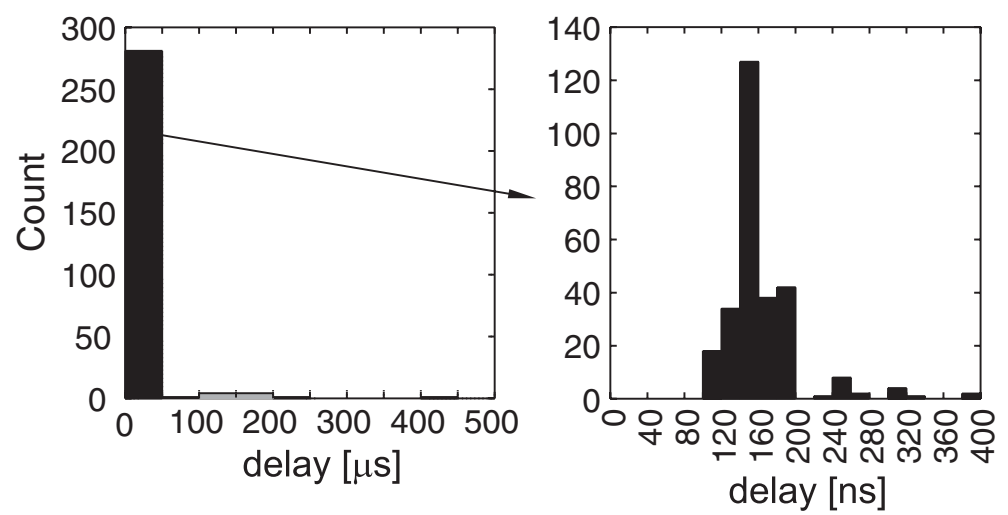

(b) $\mathrm{Ta}$



(c) stainless steel
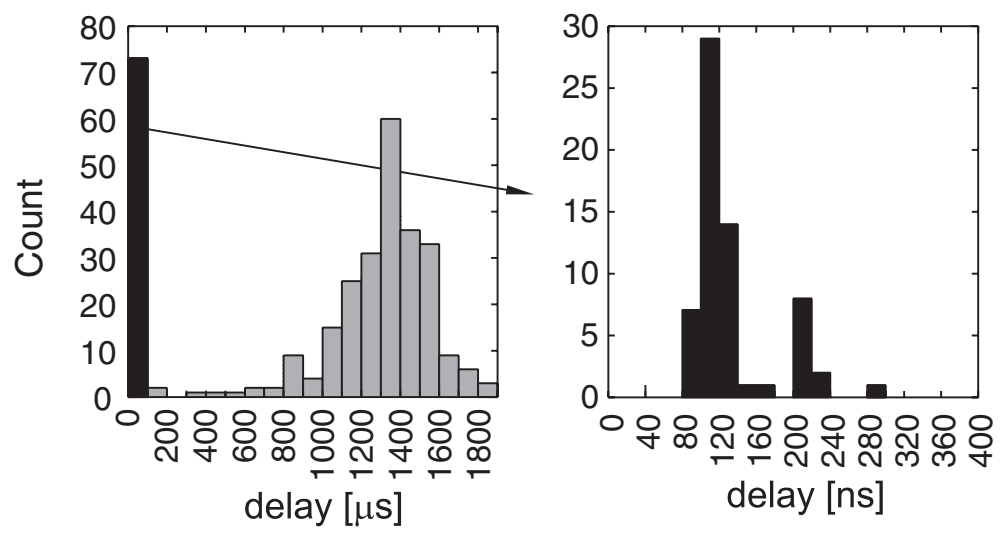

FIG. 10. Histograms of time delays with different electrode materials during conditioning experiments: (a) copper (300 breakdowns in total); (b) tantalum (250 breakdowns); (c) stainless steel (320 breakdowns). Immediate breakdowns (delays shorter than $1 \mu$ s) are displayed in black and are plotted with a higher time resolution on the right-hand side.

Figure 12 shows the outgassing of $\mathrm{H}_{2}$ measured during a breakdown rate experiment. Signals of $\mathrm{CO}, \mathrm{CH}_{4}$, and $\mathrm{CO}_{2}$ are not given here, but they are very similar. Breakdowns are easily visible, since a large rise in the $\mathrm{H}_{2}$ current is measured. Even if all breakdowns are produced at the same voltage, the heights of the peaks vary significantly from one to the other. This poor reproducibility of the sparks is a typical feature of breakdown phenomena in general. It is interesting to note that gases are also released during quiet periods when no breakdown occurs, as shown in Fig. 12(b). When the field is applied to the electrodes, electrons emitted by field emission are hitting the anode surface and release some adsorbed molecules by ESD. The presence of gas between the electrodes during quiet periods is consistent with the delay measurements presented in the previous section. According to the existing theories already 
TABLE I. Average breakdown field after conditioning and fraction of delayed breakdowns $R$ for different materials

\begin{tabular}{lcc}
\hline \hline Materials & $\bar{E}_{b}[\mathrm{MV} / \mathrm{m}]$ & $R$ \\
\hline $\mathrm{Cu}$ & 170 & 0.07 \\
$\mathrm{Ta}$ & 300 & 0.29 \\
$\mathrm{Mo}$ & 430 & 0.76 \\
Stainless steel & 830 & 0.83 \\
\hline \hline
\end{tabular}

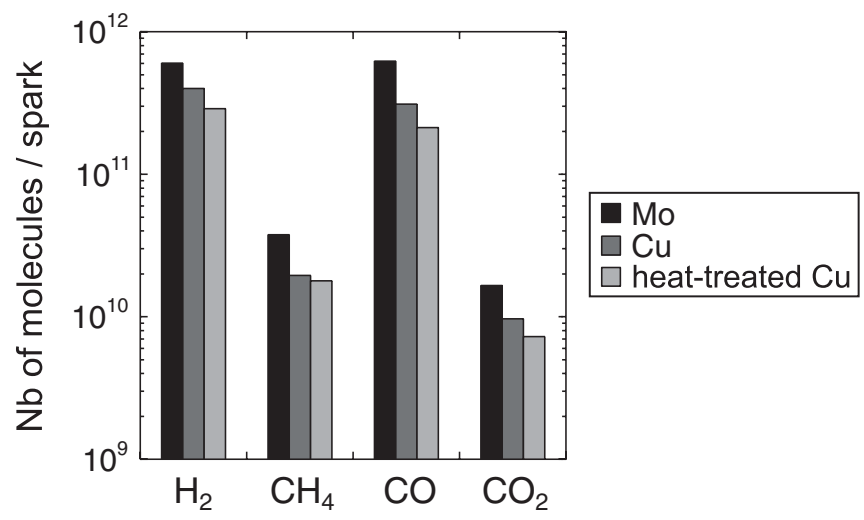

FIG. 11. Average number of molecules released per breakdown, with conditioned $\mathrm{Mo}, \mathrm{Cu}$, and heat-treated $\mathrm{Cu}$ electrodes. Data were taken at $\mathrm{BDR}=1$, at $8.4 \mathrm{kV}$ with Mo electrodes (0.95 J per breakdown), and at $7.7 \mathrm{kV}$ with $\mathrm{Cu}$ and heat-treated $\mathrm{Cu}$ electrodes $(0.8 \mathrm{~J}$ per breakdown). Values are averaged over several tens of breakdowns.

mentioned, delayed breakdowns are indeed supposed to be caused by the ionization of some gas desorbed from the surfaces. Therefore it is likely that the first breakdown of a cluster occurs with a long delay, as it is actually measured.

The outgassing level from one quiet period to the another is not necessarily the same. This is probably due to a difference in the total FE current in the beginning of the quiet period, coming either from a different $\beta$ value or a different emitting area. With a lower FE current, the number of electrons coming to the anode is lower, and so will be the outgassing level. Even if a slight increase in $\beta$ is measured just before a cluster of breakdowns (see Fig. 7), the same effect is not observed on the outgassing level [Fig. 12(b)]. Measurements of outgassing are probably less sensitive to a slight surface modification than direct measurements of $\beta$.

\section{CONCLUSION}

Although the de vacuum breakdown mechanism is not fully understood, simple experiments such as the measurement of the field enhancement factor $\beta$ can give some insight into it. It appears that the local breakdown field is the right parameter to characterize a material rather than the macroscopic breakdown field, in the sense that the values obtained during a typical conditioning experiment are less scattered and more reproducible. If one is able to know the $\beta$ value characterizing the electrode surface state at a given moment, the next macroscopic breakdown field can be predicted with a rather good precision. Knowing the evolution of $\beta$ is also crucial to understand the general pattern observed during the particular breakdown rate experiments, where a constant working field is repetitively applied to the electrodes. The unpredictability in the occurrence and in the length of the quiet periods and clusters of breakdowns directly follows from the strong variability of $\beta$ after a breakdown. Evidences that the application of a high electric field induces some surface modifications even if no breakdown occurs have also been given from $\beta$ measurements. In addition, other types of measurements such as breakdown time delays and outgassing measurements confirm the role of field emission in the breakdown (a)



(b)

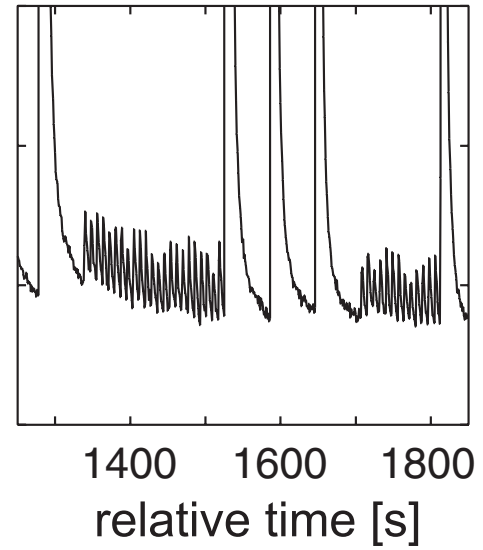

FIG. 12. $\mathrm{H}_{2}$ outgassing measured with a RGA (current for $m / e=2$ ) in a breakdown rate experiment with $\mathrm{Cu}$ electrodes, at $5 \mathrm{kV}$ $(B D R=0.1)$. A detailed view of the outgassing during two quiet periods is given in (b). After each attempt, there is a pause of 60 seconds if a breakdown occurred, and 8 seconds otherwise, to allow the pressure to recover to its nominal value. 
triggering, either directly or by secondary effects. Thus, the field enhancement factor appears as a key parameter in such breakdown experiments.

[1] W. Boyle, P. Kisliuk, and L. Germer, J. Appl. Phys. 26, 720 (1955).

[2] D. Alpert, D. Lee, E. Lyman, and H. Tomaschke, J. Vac. Sci. Technol. 1, 35 (1964).

[3] F. Charbonnier, C. Bennette, and L. Swanson, J. Appl. Phys. 38, 627 (1967).

[4] D. KennethDavies, J. Vac. Sci. Technol. 10, 115 (1973).

[5] P. Chatterton, Vacuum Breakdown, in: Electrical Breakdown in Gases, edited by J. Meek and J. Craggs (Wiley, New York, 1978).

[6] B. Juettner, Nucl. Instrum. Methods Phys. Res., Sect. A 268, 390 (1988).

[7] High Voltage Vacuum Insulation, edited by R. V. Latham (Academic Press, New York, 1995).

[8] W. Diamond, J. Vac. Sci. Technol. A 16, 707 (1998).

[9] R. Fowler and L. Nordheim, Proc. R. Soc. A 119, 173 (1928).

[10] E. Murphy and R. Good, Phys. Rev. 102, 1464 (1956).

[11] Handbook of Vacuum Arc Science and Technology: Fundamentals and Applications, edited by R.L. Boxman, D. M. Sanders, and P. J. Martin (Noyes Publications, Park Ridge, 1995).

[12] P. Slade, The Vacuum Interrupter: Theory, Design, and Application (CRC Press, Boca Raton, 2008).

[13] F. LePimpec, R. Ganter, and R. Betemps, Nucl. Instrum. Methods Phys. Res., Sect. A 574, 7 (2007).

[14] L. Laurent, Ph.D. thesis, University of California, Davis, 2002.

[15] G. Werner, Ph.D. thesis, Cornell University, Ithaca, New York, 2004.

[16] P. Wilson, in Proceedings of the 12th Advanced Accelerator Concepts Workshop, Lake Geneva, Wisconsin, 2006, AIP Conf. Proc. No. 877 (AIP, New York, 2006), p. 27.

[17] J.-P. Delahaye, in Proceedings of the 18th Particle Accelerator Conference, New York, 1999 (IEEE, New York, 1999), p. 250.

[18] G. Guignard et al., CERN Report No. CERN-2000-008, 2000.
[19] W. Wuensch, in Proceedings of the 4th Asian Particle Accelerator Conference, Indore, 2007 (RRCAT, Indore, India, 2007), p. 544.

[20] J. Rodriguez, in Proceedings of the 2007 Particle Accelerator Conference, Albuquerque, New Mexico, 2007 (IEEE, Albuquerque, New Mexico, 2007), p. 3818.

[21] W. Wuensch, in Proceedings of the 11th European Particle Accelerator Conference, Genoa, 2008 (EPS-AG, Genoa, Italy, 2008), p. 2922.

[22] M. Kildemo, Nucl. Instrum. Methods Phys. Res., Sect. A 530, 596 (2004).

[23] M. Kildemo, S. Calatroni, and M. Taborelli, Phys. Rev. ST Accel. Beams 7, 092003 (2004).

[24] T. Ramsvik, S. Calatroni, A. Reginelli, and M. Taborelli, Phys. Rev. ST Accel. Beams 10, 042001 (2007).

[25] M. Taborelli, G. Arnau-Izquierdo, S. Calatroni, S. Heikkinen, T. Ramsvik, S. Sgobba, and W. Wuensch, in Proceedings of the 2007 Particle Accelerator Conference, Albuquerque, New Mexico, 2007 (Ref. [20]), p. 2197.

[26] A. Descoeudres, T. Ramsvik, S. Calatroni, M. Taborelli, and W. Wuensch, Phys. Rev. ST Accel. Beams 12, 032001 (2009).

[27] C. Scheuerlein and M. Taborelli, Appl. Surf. Sci. 252, 4279 (2006).

[28] P. Kranjec and L. Ruby, J. Vac. Sci. Technol. 4, 94 (1967).

[29] Y. Levinsen, S. Calatroni, A. Descoeudres, M. Taborelli, and W. Wuensch, in Proceedings of the 23rd Particle Accelerator Conference, Vancouver, Canada, 2009 (IEEE, New York, 2009), p. TU5PFP012.

[30] C. Adolphsen, W. Baumgartner, K. Jobe, F. LePimpec, R. Loewen, D. McCormick, M. Ross, T. Smith, J. Wang, and T. Higo, in Proceedings of the Particle Accelerator Conference, Chicago, IL, 2001 (IEEE, New York, 2001), p. 478.

[31] G. Taylor, Proc. R. Soc. A 280, 383 (1964).

[32] S. Anders, B. Juettner, M. Lindmayer, C. Rusteberg, H. Pursch, and F. Unger-Weber, IEEE Trans. Electr. Insul. 28, 461 (1993).

[33] B. Juettner, M. Lindmayer, and G. Duening, J. Phys. D 32, 2537 (1999).

[34] G. Kartsev, G. Mesyats, D. Proskurovskii, V. Rotshtein, and G. Fursei, Sov. Phys. 15, 475 (1970).

[35] R. Ramsier and J. Yates, Surf. Sci. Rep. 12, 246 (1991).

[36] C. Benvenuti, G. Canil, P. Chiggiato, P. Collin, R. Cosso, J. Guerin, S. Ilie, D. Latorre, and K. Neil, Vacuum 53, 317 (1999). 